\title{
Two-Dimensional Mapping Separating the Acoustic Radiation Force and Streaming in
} Microfluidics

\author{
Liu, Shilei; Ni, Zhengyang; Xu, Guangyao; Guo, Xiasheng; Tu, Juan; Bruus, Henrik; Zhang, Dong
}

Published in:

Physical Review Applied

Link to article, DOI:

10.1103/PhysRevApplied.11.044031

Publication date:

2019

Document Version

Publisher's PDF, also known as Version of record

Link back to DTU Orbit

Citation (APA):

Liu, S., Ni, Z., Xu, G., Guo, X., Tu, J., Bruus, H., \& Zhang, D. (2019). Two-Dimensional Mapping Separating the Acoustic Radiation Force and Streaming in Microfluidics. Physical Review Applied, 11(4), [044031]. https://doi.org/10.1103/PhysRevApplied.11.044031

\section{General rights}

Copyright and moral rights for the publications made accessible in the public portal are retained by the authors and/or other copyright owners and it is a condition of accessing publications that users recognise and abide by the legal requirements associated with these rights.

- Users may download and print one copy of any publication from the public portal for the purpose of private study or research.

- You may not further distribute the material or use it for any profit-making activity or commercial gain

- You may freely distribute the URL identifying the publication in the public portal 


\title{
Two-Dimensional Mapping Separating the Acoustic Radiation Force and Streaming in Microfluidics
}

\author{
Shilei Liu, ${ }^{1}$ Zhengyang Ni, ${ }^{1}$ Guangyao Xu, ${ }^{1}$ Xiasheng Guo,,${ }^{1}{ }^{*}$ Juan Tu, ${ }^{1}$ Henrik Bruus, ${ }^{2}$ and \\ Dong Zhang ${ }^{1, \dagger}$ \\ ${ }^{1}$ Key Laboratory of Modern Acoustics (MOE), Department of Physics, Collaborative Innovation Center of \\ Advanced Microstructure, Nanjing University, Nanjing 210093, China \\ ${ }^{2}$ Department of Physics, Technical University of Denmark, DTU Physics Building 309, Kongens Lyngby DK-2800, \\ Denmark
}

(Received 28 November 2018; revised manuscript received 21 January 2019; published 10 April 2019)

In microscale fluids, fields of physical force and streaming play central roles in manipulating and tweezing objects, but it is difficult to disentangle and obtain accurate pictures of them. We develop a multiradius microparticle image velocimetry (MR $\mu \mathrm{PIV}$ ) protocol to solve this problem in miniaturized spaces. By using several monodisperse suspensions of spherical particles, each with its own specific particle radius, two-dimensional (2D) mapping separating the fields of radiation force and streaming is demonstrated in a microfluidic chamber driven by standing or focused surface acoustic waves, while motorized scanning is unnecessary and no special assumptions need to be made about the driving field. The results also allow the extraction of other physical parameters such as the acoustic pressure amplitude. The principle of MR $\mu$ PIV relies on a quasiequilibrium assumption for the particle motion and a linear dependence of the field force on particle volume. Therefore, it is also applicable to tweezing techonologies using optical, dielectrophoretic, and magnetic forces, constituting an extension of the PIV technique impactful for microscale physics in general.

DOI: 10.1103/PhysRevApplied.11.044031

\section{INTRODUCTION}

Physical (e.g., acoustic, optical, electric, and magnetic) fields exerted on microscale fluids have proven efficient in noncontact manipulation of micro/nano objects in physics, chemistry, and biology [1-6]. In these processes, particle manipulation relies on controlling external forces and streaming drag by regulating the fields [7-9] and particle properties $[10,11]$. For example, the acoustic radiation force (ARF) and the acoustic streaming (AS) independently or jointly play dominant roles in acoustofluidics $[11,12]$. A critical diameter can be identified for spherical particles of a specific material, above which particle motion crosses from streaming dominated to radiation dominated $[12,13]$.

Calibration of these force fields and the streaming is difficult. Traditional methods like using force sensors fail due to the miniaturized dimension of the devices. To measure the field force on a single particle, protocols have been proposed based on its static or quasistatic balance with other forces, typically optical [14], electric [15], and gravitational forces [16], while motorized scanning is necessary to get a full landscape of the field. For streaming,

\footnotetext{
*guoxs@nju.edu.cn

†dzhang@nju.edu.cn
}

readouts from microparticle image velocimetry ( $\mu$ PIV) $[17,18]$ using micron or submicron beads are effective in both two dimensions [19] and three dimensions [20,21]. However, in determining either the force or the streaming, the other one is usually neglected [15], inducing calibration inaccuracies. Currently, we lack a technique that provides a full picture of force and streaming in microscale fluids.

Stable actuation fields usually induce steady-state fields of force and streaming. Shortly after the fields are established, the suspended particles execute so-called quasiequilibrium motions due to the dynamic equilibrium between the field force and the viscous drag from the fluid flow [12,19]. Based on acoustofluidics, we here report a multiradius $\mu \mathrm{PIV}$ (MR $\mu \mathrm{PIV}$ ) protocol to extract the force and streaming fields from $\mu$ PIV measurements using the size-dependent acoustophoretic response of tracer particles having different but well-defined radii. The acoustic pressure amplitude and the electroacoustic scaling factor (EASF), defined as the ratio of the amplitudes of the acoustic pressure and the driving voltage and reflecting the actuation efficiency [19], is also obtained for standing acoustic fields. Analogous to the gravitational field, the ARF is represented by the field of particle acceleration (FOA); and for spherical particles of the same material, the FOA is independent of their sizes. 


\section{THEORY AND PROTOCOL}

We prepare four different dilute and monodisperse suspensions of spherical polystyrene tracer particles of density $\rho_{p}=1050 \mathrm{~kg} / \mathrm{m}^{3}$ and compressibility $\kappa_{p}=249 \mathrm{TPa}^{-1}$, and with respective radii of $r=(0.49 \pm 0.02),(1.50 \pm$ $0.04),(2.52 \pm 0.04)$, and $(4.90 \pm 0.05) \mu \mathrm{m}$ (nominal $r=$ $0.5,1.5,2.5,5.0 \mu \mathrm{m}$, Microparticles $\mathrm{GmbH}$ ). The fluid medium is water with density $\rho_{m}=997 \mathrm{~kg} / \mathrm{m}^{3}$, compressibility $\kappa_{m}=448 \mathrm{TPa}^{-1}$, and viscosity $\eta=0.89 \mathrm{mPas}$. The suspensions are injected into the microchannel and an acoustic field is established. The resulting acoustic radiation force $\boldsymbol{F}_{r}$ acting on a given particle is proportional to its volume $V_{p}=\frac{4}{3} \pi r^{3}$ [22], so the corresponding acceleration is $\boldsymbol{a}_{r}=\left(\rho_{p} V_{p}\right)^{-1} \boldsymbol{F}_{r}$. The Stokes drag force on a particle moving with velocity $\boldsymbol{v}_{p}$ is $\boldsymbol{F}_{d}=6 \pi \eta r\left(\boldsymbol{v}_{m}-\right.$ $\boldsymbol{v}_{p}$ ), where $\boldsymbol{v}_{m}$ is the local fluid velocity, here originating from the AS. The corresponding acceleration is $\boldsymbol{a}_{d}=$ $B\left(\boldsymbol{v}_{m}-\boldsymbol{v}_{p}\right) r^{-2}$ with $B=9 \eta /\left(2 \rho_{p}\right)$. The overall particle acceleration is

$$
\boldsymbol{a}_{p}=\boldsymbol{a}_{r}+B\left(\boldsymbol{v}_{m}-\boldsymbol{v}_{p}\right) r^{-2} .
$$

In Ref. [19], we presented a $\mu$ PIV experiment to measure the acoustic pressure amplitude in a microchamber driven by standing surface acoustic waves (SAWs). The same system and chip design are used and illustrated in Fig. 1. The substrate is a 2-in.-diameter $128^{\circ} Y-X$ lithium niobate $\left(\mathrm{LiNbO}_{3}\right)$ wafer (with thickness $500 \mu \mathrm{m}$ ). The doublelayered interdigital transducers (IDTs) $(\mathrm{Cr} / \mathrm{Au}, 50 \AA / 2200$ $\AA$ ) are deposited on the substrate through lithography. A $1 \times 1-\mathrm{cm}$ block bonded to the substrate is fabricated using polydimethylsiloxane (PDMS, Sylgard 184, Dow Corning) through soft lithography and mold replication, in which a $2 \times 2$-mm chamber (with height $220 \mu \mathrm{m}$ ) is embedded. Fluorescent beads injected into the chamber are illuminated with a 532-nm laser beam and the emitted 610-nm fluorescence is captured for PIV analysis. In the experiments, we observe the migration of monodispersed beads in the four suspensions diluted enough to ensure negligible particle-particle interactions. Two counterpropagating SAWs in the horizontal $x-y$ plane establish a standing wave along the $y$ axis in the chamber. The IDTs are connected in parallel and excited through a 55-dB power amplification of a $13.45-\mathrm{MHz}$ sinusoidal signal. As a polystyrene particle in water has a positive contrast factor $\Phi$ [12], they aggregate around the nodal lines parallel to the $x$ direction. This result is shown in Appendix A, where the particles in the suspensions are seen to have formed striped patterns within different time periods, and the crossover from ASto ARF-dominated motion can be inferred as $r$ increases from 0.5 to $5.0 \mu \mathrm{m}$.

In $\mu$ PIV analysis, a readout map represents the velocity of all particles across the field of view in a dilute suspension. Hence, an Eulerian velocity field $\boldsymbol{v}_{p}(x, y)$ is

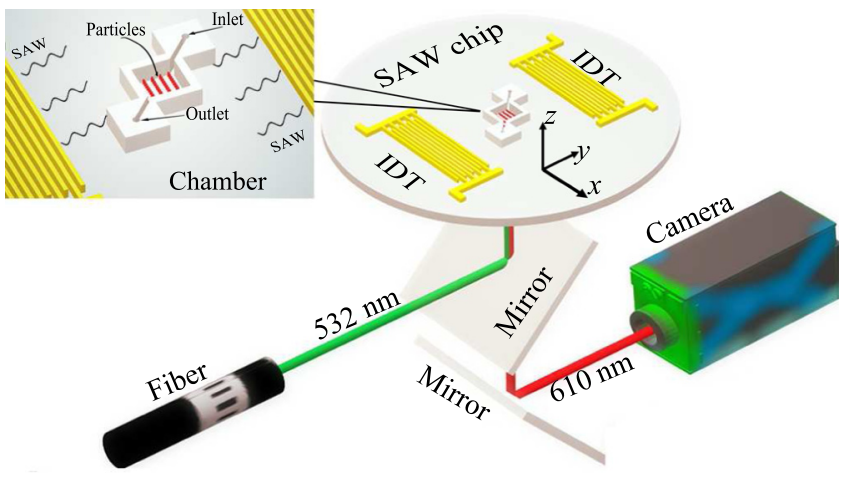

FIG. 1. A scheme of the experimental setup and chip design.

introduced to describe the velocity of a particle located at $(x, y)$. Similarly, $\boldsymbol{v}_{m}(x, y)$ and $\boldsymbol{a}_{r}(x, y)$ describe the AS field and the FOA, while $\boldsymbol{a}_{p}(x, y)$ represents the particle acceleration in Eq. (1). When treated as an Eulerian field, $\boldsymbol{a}_{p}(x, y)$ is the sum of a local and an advective term,

$$
\boldsymbol{a}_{p}=\partial_{t} \boldsymbol{v}_{p}+\left(\boldsymbol{v}_{p} \cdot \nabla\right) \boldsymbol{v}_{p}
$$

where $\partial_{t}$ is the time derivative and $\nabla$ is the spatial gradient operator. Since the speeds of the largest particles $(r=5 \mu \mathrm{m})$ in the experiments never exceed $200 \mu \mathrm{m} / \mathrm{s}$, the particle Reynolds number is less than $5 \times 10^{-4}$ and, consequently, the inertial effects are neglected. This process is also seen in Fig. 2, where surface plots of the $x$-averaged $y$ component $\boldsymbol{v}_{p, y}(y, t)$ of the particle velocity field $\boldsymbol{v}_{p}$ are shown for each of the four suspensions as a function of $y$ and time $t$. In each plot, a short transient phase is followed by a long quasiequilibrium phase, in which we can assume

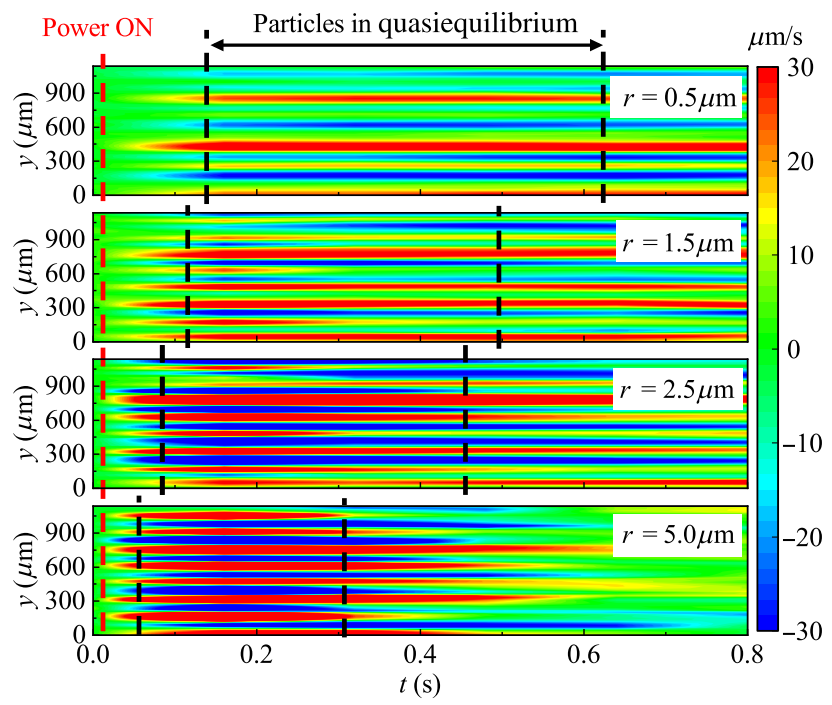

FIG. 2. Color plot of the $y$ component $\boldsymbol{v}_{p, y}$ of the particle velocity averaged along the $x$ direction as a function of time and $y$ coordinate for particles with radius $r=0.5,1.5,2.5$, and $5.0 \mu \mathrm{m}$ for the SAW-driving voltage $V_{\mathrm{pp}}=55 \mathrm{mV}$. 
a vanishing local acceleration, $\partial_{t} \boldsymbol{v}_{p}=\mathbf{0}$ [12]. Hence, in Eq. (2), the particle acceleration is dominated by the advective term. It should also be mentioned that, in Fig. 2, particles of larger size enter quasiequilibrium quicker but stay shorter. The reason is because the ARF and the Stokes drag are respectively proportional to $r^{3}$ and $r$. For larger particles, they accelerate faster and are balanced at higher speeds due to the dominance of ARF. For smaller particles, deceleration due to streaming drag becomes more significant, resulting in slower movements.

In principle, the AS field $\boldsymbol{v}_{m}$ and the FOA $\boldsymbol{a}_{r}$ can be determined according to Eqs. (1) and (2) once $\boldsymbol{v}_{p}(x, y)$ corresponding to particles of two different radii have been extracted from PIV. However, to minimize the measurement uncertainties, we use more particle sizes, and $\boldsymbol{v}_{m}(x, y)$ and $\boldsymbol{a}_{r}(x, y)$ are obtained from an overdetermined matrix equation,

$$
\begin{gathered}
\overline{\bar{A}} \cdot \bar{X}=\bar{b}, \quad \text { with } \overline{\bar{A}}=\left[\begin{array}{cc}
1 & B r_{(1)}^{-2} \\
1 & B r_{(2)}^{-2} \\
\vdots & \vdots \\
1 & B r_{(n)}^{-2}
\end{array}\right], \quad \bar{X}=\left[\begin{array}{c}
\boldsymbol{a}_{r}(x, y) \\
\boldsymbol{v}_{m}(x, y)
\end{array}\right], \\
\bar{b}=\left[\begin{array}{c}
\left(\boldsymbol{v}_{p(1)}(x, y) \cdot \nabla\right) \boldsymbol{v}_{p(1)}(x, y)+B r_{(1)}^{-2} \boldsymbol{v}_{p(1)}(x, y) \\
\left(\boldsymbol{v}_{p(2)}(x, y) \cdot \nabla\right) \boldsymbol{v}_{p(2)}(x, y)+B r_{(2)}^{-2} \boldsymbol{v}_{p(2)}(x, y) \\
\vdots \\
\left(\boldsymbol{v}_{p(n)}(x, y) \cdot \nabla\right) \boldsymbol{v}_{p(n)}(x, y)+B r_{(n)}^{-2} \boldsymbol{v}_{p(n)}(x, y)
\end{array}\right] .
\end{gathered}
$$

Here, the index $(i)$ refers to particles with radius $r_{(i)}$. In applying the algorithm, PIV data corresponding to a single copy of solutions of each particle size are used, inducing $n=4$. Solutions $\bar{X}$ of Eq. (3) are obtained through an ordinary least-squares scheme, minimizing the sum of squared residuals (SSR) $\|\overline{\bar{A}} \cdot \bar{X}-\bar{b}\|$ with respect to $\bar{X}$. Specifically, $\|\overline{\bar{A}} \cdot \bar{X}-\bar{b}\| / \bar{b}_{\max }$ is defined as the relative SSR, in which $\bar{b}_{\max }$ is the maximum value in $\bar{b}$. The averaged relative SSR is found to be approximately 0.22 . Based on PIV measurements of $\boldsymbol{v}_{p}(x, y)$ of the four suspensions at $t=0.28 \mathrm{~s}$, the AS field and the FOA are determined. For the choice of $\mu$ PIV parameters, see Fig. 7 in Appendix B and Fig. 8 in Appendix C. Each measurement is repeated three times with new copies of particle solutions and the obtained results are finally averaged over the three repetition experiments. Hence, dashed variables in Eq. (3) represent averaged data. Possible $x$ averaging is carried out for the individual experiments before the three-experiment averaging.

\section{RESULTS AND DISCUSSIONS}

For the driving voltage $V_{\mathrm{pp}}=55 \mathrm{mV}$, the $y$ component of the AS field is given in Fig. 3(a). The fluid velocity field is a little disordered, as observed in SAW [23] and bulk acoustic wave (BAW) [12] acoustofluidics. Streaming rolls are insignificant in the field, since they exist mostly in planes perpendicular to the $x-y$ plane $[20,23]$ and since the field is away from the channel boundaries. The motion of the $0.5-\mu \mathrm{m}$ particles is entirely due to AS $[20,24]$, and $\boldsymbol{v}_{p}(x, y)=\boldsymbol{v}_{m}(x, y)$ is expected. With three replicate experiments, $v_{p, y}(x, y)$ of $0.5-\mu \mathrm{m}$ particles and $v_{m, y}(x, y)$ determined from MR $\mu \mathrm{PIV}$ are averaged along the $x$ direction and presented as functions of $y$ in Fig. 3(c) for comparison. A good agreement is observed between the two, with a correlation coefficient CORR $=0.99$.

The $y$ component of the FOA in Fig. 3(b) shows that the ARF pushed particles toward the nodal lines located every half wavelength, $\frac{1}{2} \lambda=150 \mu \mathrm{m}$. Acoustophoretic motions of the $5.0-\mu \mathrm{m}$ particles are almost entirely ARF governed $[20,25,26]$. The FOA $\boldsymbol{a}_{r}(x, y)$ determined by MR $\mu$ PIV is independent of the particle size and should be equivalent to $\boldsymbol{a}_{r}^{\prime}(x, y)=\left[\boldsymbol{v}_{p}(x, y) \cdot \nabla\right] \boldsymbol{v}_{p}(x, y)+B r^{-2} \boldsymbol{v}_{p}(x, y)$ of $5.0-\mu \mathrm{m}$ particles, in which contribution from the AS is neglected. Maps of $a_{r, y}(x, y)$ and $a_{r, y}^{\prime}(x, y)$ are averaged along $x$ and plotted as functions of $y$ in Fig. 3(d). Slight discrepancy is observed between the two and the low value CORR $=0.93$ is partly due to AS. Specifically, $\boldsymbol{a}_{r}^{\prime}(x, y)$ can be underestimated where the particle acceleration is reduced by local AS. Therefore, even for large particles, neglect of AS, as was done in previous models [19,27], can induce inaccuracy in acoustic calibrations as pointed out in Ref. [12].

To study the joint effects of ARF and AS in acoustophoresis, velocity fields $\boldsymbol{v}_{p}(x, y)$ corresponding to each particle size are decomposed into weighted sums of contributions from ARF and AS through an ordinary leastsquares (OLS) scheme (see Appendix D for details). The weighting coefficients $\alpha$ for ARF and $\beta$ for AS are listed in Table I. With the increase of $r$, there is a monotonic growth in $\alpha$ and decline in $\beta$, demonstrating the evolution from AS-dominated motion for $r=0.5 \mu \mathrm{m}$ to ARF-dominated motion for $r=5.0 \mu \mathrm{m}$. A characteristic particle radius $r_{c}$, separating AS-dominated and ARF-dominated particle motions, can be defined by assuming the force balance $\boldsymbol{F}_{r}=\boldsymbol{F}_{d}$. Here, we find $r_{c}=1.56 \mu \mathrm{m}$, agreeing well with the prediction $r_{c}=1.3 \mu \mathrm{m}$ obtained by using the model of Barnkob et al. [12]. It is noteworthy that the $0.5-\mu \mathrm{m}$ particles are undergoing streaming-dominated movements and the ARF acting on them are quite small. In this case, the corresponding weighting factor $\alpha$ should be very close to zero. Therefore, the negative sign should be attributed to experimental measurement errors.

In ideal standing-SAW acoustophoresis, the particle acceleration $\boldsymbol{a}_{r}$ due to the ARF should be sinusoidal along the wave vector. The $y$ component of $\boldsymbol{a}_{r}(x, y)$ can be written as [22]

$$
\boldsymbol{a}_{r, y}(x, y)=\frac{k \Phi}{\rho_{p}} \frac{1}{4} \kappa_{m} p_{0}^{2} \sin (2 k y),
$$


$y$-component AS $v_{m, y}(x, y)(\mu \mathrm{m} / \mathrm{s}) \quad y$-component FOA $a_{r, y}(x, y)\left(\mathrm{m} / \mathrm{s}^{2}\right)$

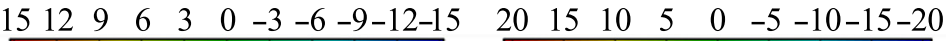

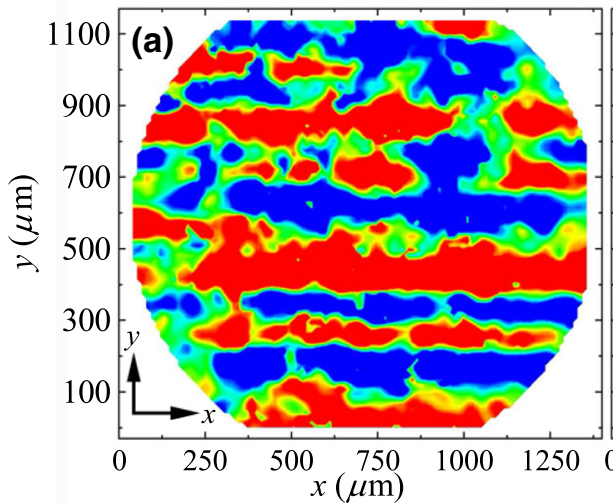

FIG. 3. The $y$-component fields of (a) the AS and (b) the FOA determined from MR $\mu \mathrm{PIV}$. (c) The $x$ averaged $v_{m, y}(x, y)$ from $\mathrm{MR} \mu \mathrm{PIV}$ and $v_{p, y}(x, y)$ from $\mu \mathrm{PIV}$ using 0.5 $\mu \mathrm{m}$ particles. (d) The $x$-averaged $a_{r, y}(x, y)$ from MR $\mu$ PIV and $a_{r, y}^{\prime}(x, y)$ from $\mu$ PIV using $5-\mu \mathrm{m}$ particles. The shadowed areas in (c),(d) are errors. Driving $V_{\mathrm{pp}}=55 \mathrm{mV}, t=0.28 \mathrm{~s}$.

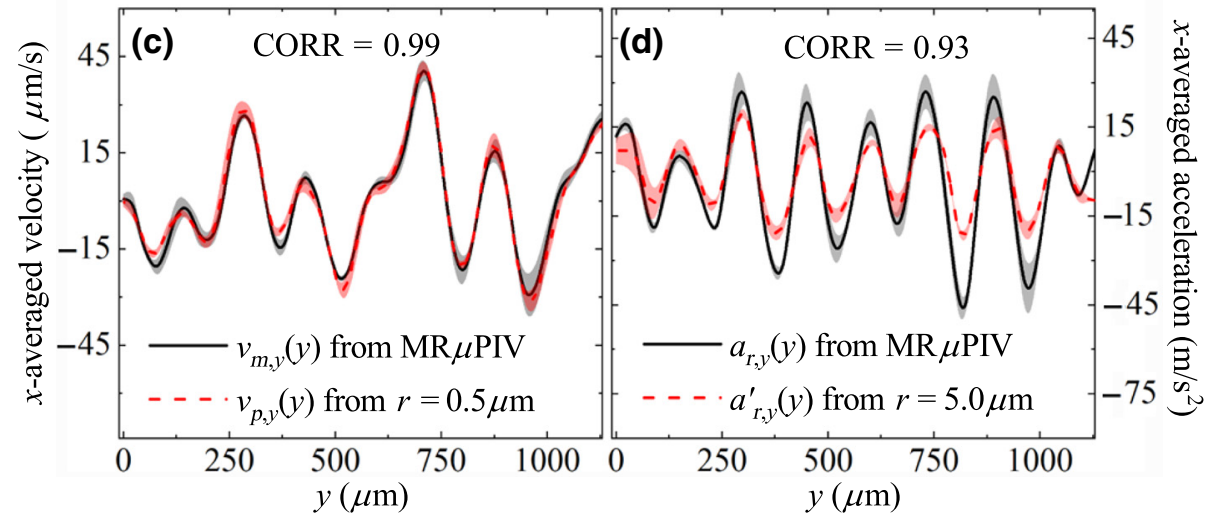

where $k=2 \pi / \lambda$ and $p_{0}$ is the acoustic pressure amplitude. The $x$-averaged acceleration $\boldsymbol{a}_{r, y}(y)$ shown in Fig. 3(d) and obtained from MR $\mu$ PIV is fitted in Fig. 4(a) with $\chi \sin (2 k y+\psi)+$ const, in which $\chi=\frac{1}{4} \kappa_{m} p_{0}^{2} k \Phi / \rho_{p}$, and $p_{0}=142 \mathrm{kPa}$ is obtained for $V_{\mathrm{pp}}=55 \mathrm{mV}$. Considering the electroacoustic coupling to be linear, $p_{0}$ should be proportional to $V_{\mathrm{pp}}$, i.e., $p_{0}=\zeta V_{\mathrm{pp}}$ with $\zeta$ being the EASF $[19,27]$. In Fig. 4(b), we plot the measured pressure amplitude $p_{0}$ as a function of the driving voltage $V_{\mathrm{pp}}$ from 55 to $75 \mathrm{mV}$ in steps of $5 \mathrm{mV}$. By linear fitting, we obtain the EASF $\zeta=2.69 \mathrm{MPa} / \mathrm{V}$. In agreement with this MR $\mu$ PIV value, we also obtain the value $\zeta=2.48 \mathrm{MPa} / \mathrm{V}$, only $8 \%$ lower, from a finite-element (FE) simulation using the amplitude of the SAW measured by a laser vibrometer as the driving boundary condition; see Fig. 9 in Appendix E.

In previous measurements of $p_{0}$ in acoustophoresis of particles with $r>3 \mu \mathrm{m}$, the AS was neglected [19,27], while for studies with smaller particles, it was taken into account [12]. Here, we study more closely when AS must be taken into account. If we assume that the Stokes drag $\boldsymbol{F}_{d \text {, Eu }}=-6 \pi \eta r \boldsymbol{v}_{p}$ arises solely from the Eulerian particle velocity, it follows from quasiequilibrium $\boldsymbol{F}_{d, \mathrm{Eu}}+$ $\boldsymbol{F}_{r}=0$ that the ARF is $\hat{\boldsymbol{a}}_{r}=B r^{-2} \boldsymbol{v}_{p}$. Given that $\boldsymbol{a}_{r}=$ $B r^{-2}\left(\boldsymbol{v}_{p}-\boldsymbol{v}_{m}\right)+\boldsymbol{a}_{p}$ by Eq. (1), the accuracy of the previous method depends on $\boldsymbol{a}_{p}$ and $\boldsymbol{v}_{m}$ being negligible relative to $\boldsymbol{a}_{r}$ and $\boldsymbol{v}_{p}$, respectively. In our experiments, we observe $\boldsymbol{a}_{p} \approx 10^{-6} \mathrm{~m} / \mathrm{s}^{2}$, while the maximum $\boldsymbol{a}_{r, y} \approx 40 \mathrm{~m} / \mathrm{s}^{2}$ in Fig. 3(d). The fact that $\boldsymbol{a}_{p}$ is negligibly small might explain the phenomenon in Fig. 2 that $\partial_{t} \boldsymbol{v}_{p}$ becomes zero shortly after the acoustic field is turned on. On the other hand, the streaming velocity $v_{m}$ is as high as $45 \mu \mathrm{m} / \mathrm{s}$ in Fig. 3(c), comparable with $v_{p} \lesssim 150 \mu \mathrm{m} / \mathrm{s}$ in Fig. 2. Consequently, neglect of AS could induce noticeable errors in field mapping.

However, $\boldsymbol{a}_{r}-\hat{\boldsymbol{a}}_{r} \approx-\mathrm{Br}^{-2} \boldsymbol{v}_{m}$ decreases with increased $r$, and for sufficiently large particles, their motion is almost entirely due to ARF, and the EASF $\zeta$ (and $p_{0}$ ), measured by our previous protocol [19], should be equivalent to that obtained here, $\zeta=2.69 \mathrm{MPa} / \mathrm{V}$. To verify this, the same data-processing scheme is carried out, and $\zeta=5.12,3.45$, and $2.70 \mathrm{MPa} / \mathrm{V}$ are found for $1.5,2.5$, and $5.0-\mu \mathrm{m}$ particles, respectively. There is no doubt that larger particles might give a $\zeta$ value closer to $2.69 \mathrm{MPa} / \mathrm{V}$, but related experiments are hard to carry out since acoustic scattering

TABLE I. Weighting factors $\alpha$ for ARF and $\beta$ for AS.

\begin{tabular}{lcccc}
\hline \hline$r(\mu \mathrm{m})$ & 0.5 & 1.5 & 2.5 & 5.0 \\
\hline$\alpha$ & $-0.04 \pm 0.08$ & $0.61 \pm 0.09$ & $0.80 \pm 0.09$ & $0.94 \pm 0.04$ \\
$\beta$ & $0.89 \pm 0.10$ & $0.54 \pm 0.05$ & $0.40 \pm 0.27$ & $0.15 \pm 0.12$ \\
\hline \hline
\end{tabular}



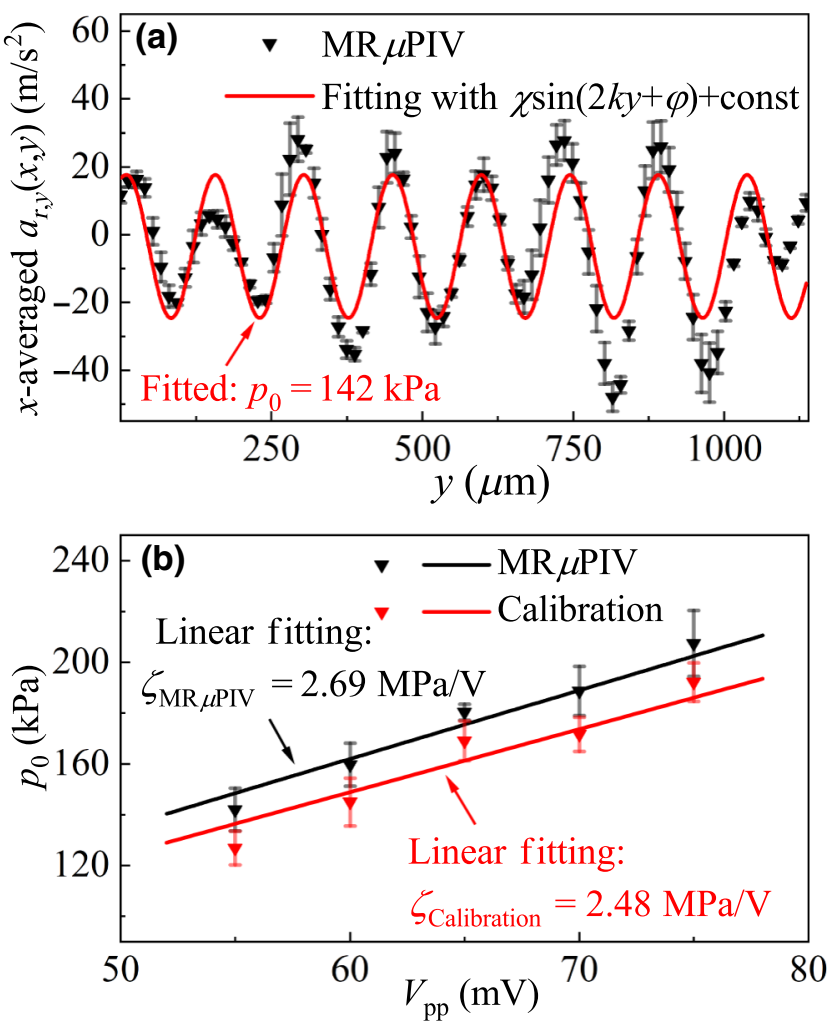

FIG. 4. (a) Fitting of $a_{r, y}(x, y)$ (black triangles) with $\chi \sin (2 k y+\psi)+$ const (red line) at $V_{\mathrm{pp}}=55 \mathrm{mV}$, leading to $p_{0}=142 \mathrm{kPa}$. (b) The EASF $\zeta$ determined by linear fits of $p_{0}$ vs. $V_{\text {pp }}$ obtained by MR $\mu$ PIV (black) and by a laser vibrometer combined with FE analysis (red).

can become intense [22]. It should be mentioned that, in the experiments, microscopic observations are carried out away from the cavity boundaries and the boundary streaming effects should be insignificant. But in principle, the proposed method is valid as soon as the streaming patterns are stable, or when regular $\mu \mathrm{PIV}$ measurements are effective.

Spatial distributions of microscale physical fields, including those of radiation force and streaming, are crucial for manipulating and tweezing micro/nanoparticles. But even with the same theoretical design, the experimentally observed phenomenon could differ due to reasons such as the ignorance of other physical factors. Therefore, field mapping and calibration is essential for the development of these technologies and is of course necessary for their industrial deployment.

Similar to acoustic force measurement using optical tweezers, the MR $\mu$ PIV approach benefits from making no assumptions about the pressure field [14]. For example, Eqs. (1)-(3) do not rely on the excitation of SAWs or the establishment of a standing field. Hence, the current technique is also applicable to acoustofluidics driven by other waves such as BAWs or traveling acoustic waves.
For example, application of this technique in microfluidics actuated with focused SAWs is also demonstrated here. The focused field is generated from an arc-shaped IDT, which includes 33 pairs of electrodes deposited on a $\mathrm{LiNiO}_{3}$ substrate (with thickness $500 \mu \mathrm{m}$ ) and has an aperture angle of $110^{\circ}$. The axial direction of the SAW beam is along the $-y$ axis. The other experimental parameters are the same as in the previous experiments, while all measurements are repeated three times, giving the MR $\mu$ PIV results presented in Fig. 5. It should be mentioned that amplitudes rather than the $x$ or $y$ components of the AS and FOA are used here for analysis.

From the AS and FOA patterns shown in Figs. 5(a) and 5 (b), acoustic energy is focused to a predefined region, while side lobes of the beam cause standinglike patterns along the radial direction over a very localized area. The two-dimensional (2D) streaming field is averaged along the $y$ direction across a strip domain $( \pm \lambda / 12$ from the focal center), which is recorded as $v_{m}(x)$ and shown as a function of $x$ in Fig. 5(c). For comparison, the velocity field of the $0.5-\mu \mathrm{m}$ particles, $v_{p}(x)$, is also given. The excellent agreement between the two, with a CORR $=0.99$, demonstrates the feasibility of applying MR $\mu$ PIV for focused ultrasound calibrations. The FOA of the focused field in Fig. 5(b) shows that particles accelerated much faster around the focal region. Using a protocol similar to that used in obtaining Fig. 3(d), maps of $\boldsymbol{a}_{r}$ (from MR $\mu \mathrm{PIV}$ ) and $\boldsymbol{a}_{r}^{\prime}$ (from 5.0- $\mu \mathrm{m}$ particles) across the above-mentioned strip area are averaged along the $y$ direction and presented as functions of $x$ in Fig. 5(d). It is observed that the two averaged profiles are roughly consistent, with a CORR $=0.79$. The hardly negligible difference between the two provides powerful backing to MR $\mu$ PIV rather than undermining its reliability and can be explained as follows. In obtaining $\boldsymbol{a}_{r}^{\prime}$ using $5.0-\mu \mathrm{m}$ particles, the fluid velocity $\boldsymbol{v}_{m}$ is assumed small enough to be neglected. This is relatively valid in the plane standing wave field case, where the maximum amplitude of the $y$ component $\boldsymbol{v}_{m}$ is about $40 \mu \mathrm{m} / \mathrm{s}$. However, in the focused field, the fluid velocity is much higher around the neighborhood of the focal center, which is close to $200 \mu \mathrm{m} / \mathrm{s}$. Therefore, disparity between the two curves in Fig. 5(d) actually indicates that it becomes inappropriate to consider the $5.0-\mu \mathrm{m}$ particles to be totally radiation dominated. As a conclusion, the observations here further demonstrate the importance of accounting for AS in the process of field mapping.

In general, MR $\mu$ PIV applies when (a) the particles undergo quasiequilibrium motions and (b) the force exerted on a particle is linearly dependent on the particle volume. The former can easily be fulfilled as soon as the force field is stable. The latter condition is also valid for the optical force [4], the dielectrophoretic force [5], and the magnetic moment [6] at varied scales. Therefore, MR $\mu$ PIV should be applicable to microfluidics combined with these physics, giving acceleration fields due to the forces and the 

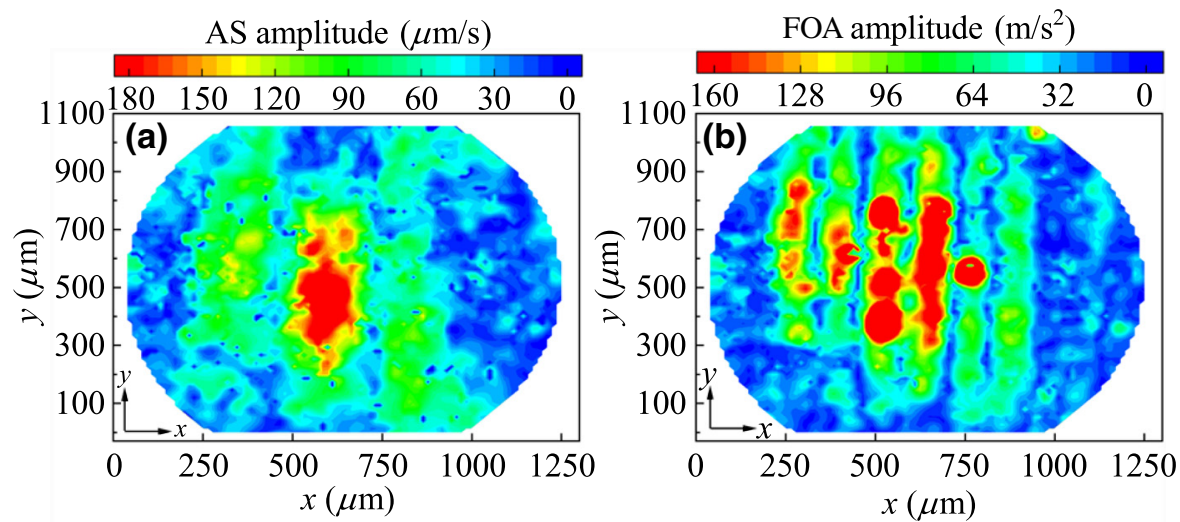

FIG. 5. The amplitude of (a) AS and (b) FOA patterns actuated by a focused SAW beam. (c) Comparison between the $y$-averaged AS amplitude determined from $\mathrm{MR} \mu \mathrm{PIV}$ and the corresponding velocity amplitude of the $0.5-\mu \mathrm{m}$ particles. (d) Comparison between the $y$ averaged FOA amplitude determined from $\mathrm{MR} \mu \mathrm{PIV}$ and the acceleration amplitude of 5.0- $\mu \mathrm{m}$ particles.
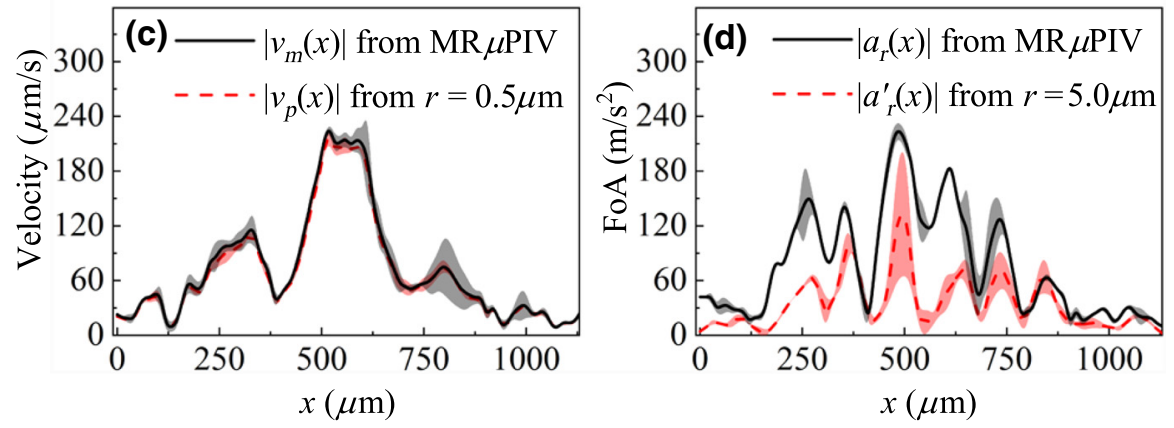

streaming fields. During the applications, when analytical predictions of other uniformly distributed field parameters (e.g., the pressure amplitude of planar waves) is available, they can also be calibrated with this technique. It should be mentioned that MR $\mu$ PIV could suffer from limitations when used to characterize inertial lifts in microchannels, since the lift force is proportional to the fourth power of the particle radius [28].

The beauty of MR $\mu$ PIV lies in mapping the disentangled fields of force and streaming simultaneously. Neglecting either of the two in determining the other one can induce unpredictable inaccuracies, as is indicated from Figs. 3(c) and 3(d). This conclusion should also apply when the driving physics is switched to other forms.

\section{CONCLUSIONS}

Demonstrated using acoustofluidic devices, the proposed MR $\mu$ PIV technique can help to disentangle the fields of ARF and AS and achieve individual mapping of them, while other uniformly distributed field parameters like the acoustic pressure amplitude can also be determined. The field separation relies on stable physical actuation, quasiequilibrium motions of particles in acoustophoresis, and the linear dependence on particle volume for ARF. It therefore can be applied to the counterparts of acoustofluidics, e.g., optofluidics and magnetofluidics. Capable of achieving 2D separated mapping without motorized scanning, the MR $\mu$ PIV protocol provides more comprehensive and accurate physical views in microscale fluids than the conventional $\mu$ PIV technique.

\section{ACKNOWLEDGMENTS}

This work is supported by the National Natural Science Foundation of China (Grants No. 81627802, No. 11774166, No. 11774168, No. 11874216, and No. 11674173), the Qinglan Project, and the Fundamental Research Funds for the Central Universities.

S. Liu and Z. Ni contributed equally to this work.

\section{APPENDIX A: PARTICLE ACOUSTOPHORESIS}

In the 13.45-MHz standing field (driving $V_{\mathrm{pp}}=55 \mathrm{mV}$ ) built along the $y$ direction, PIV-observed particle patterns and corresponding velocity maps are presented in Fig. 6 . Because of AS, the $0.5-\mu \mathrm{m}$ particles are not successfully patterned. In the 1.5- and $2.5-\mu \mathrm{m}$ cases, the particles are in transition between ARF- and AS-dominated movements [12]. The $1.5-\mu \mathrm{m}$ particles move slower than both the 0.5 and $2.5-\mu \mathrm{m}$ ones, which coincides with the observations reported in Ref. [24]. The 5.0- $\mu \mathrm{m}$ particles are undergoing ARF-dominated motions [29] and approach the field node lines much faster than others.

\section{APPENDIX B: TIME TO CARRY OUT MR $\mu$ PIV ANALYSIS}

As illustrated in Fig. 1, particles are in dynamic equilibrium shortly after the acoustic field is turned on until the acoustophoresis process is completed. MR $\mu$ PIV analysis, which benefits from $\partial_{t} \boldsymbol{v}_{p}=\mathbf{0}$, is carried out during this process at $t=0.28 \mathrm{~s}$. For comparison, data acquired at $t=0.14,0.42$, and $0.56 \mathrm{~s}$ are also used for analysis. The 


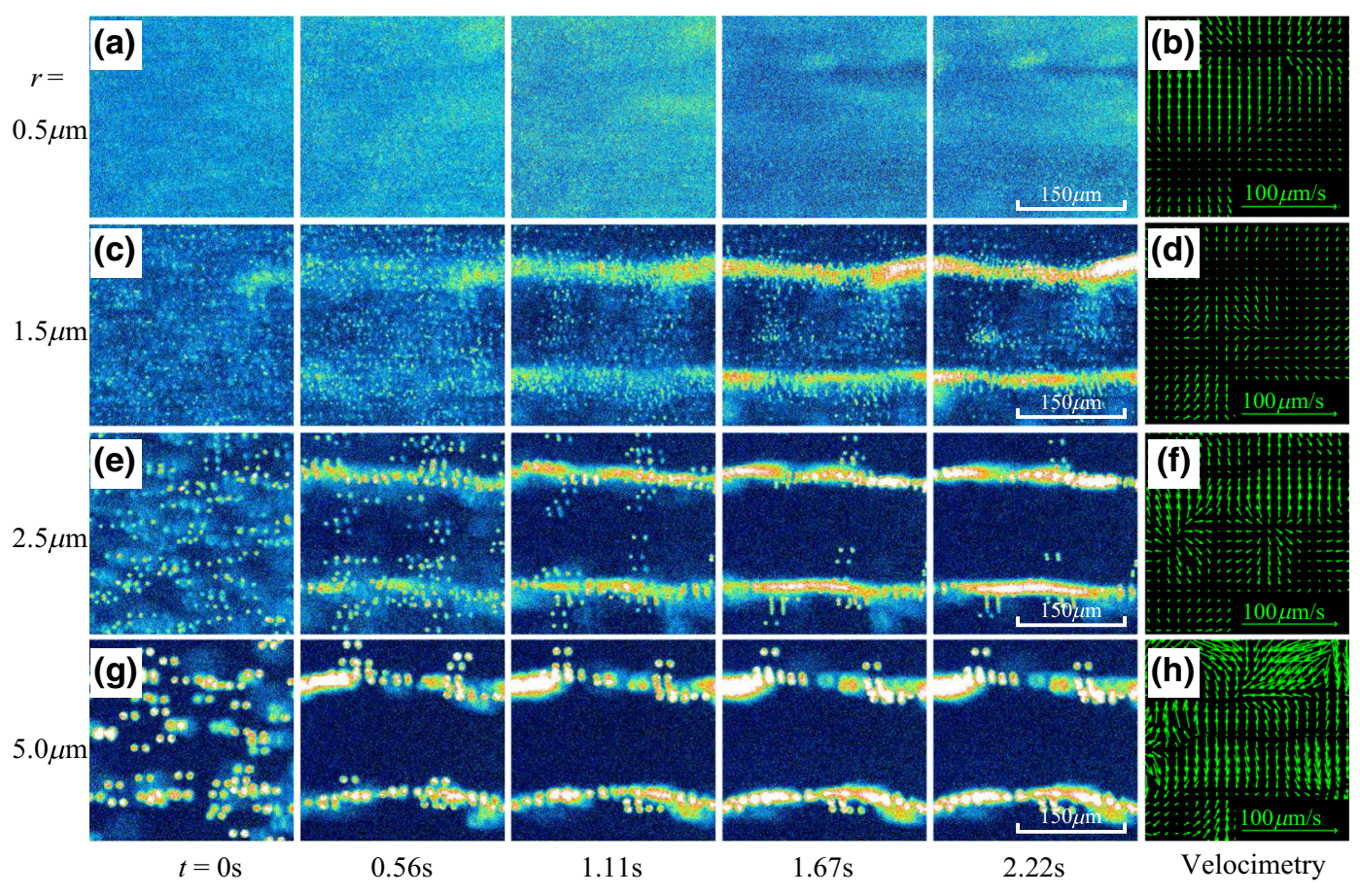

FIG. 6. Snapshots (columns 1-5) and corresponding velocimetry results (column 6) of particle motions in an acoustophoresis channel driven by $13.45-\mathrm{MHz}$ standing SAWs. Driving $V_{\mathrm{pp}}=55 \mathrm{mV}$.

results in Fig. 7 include (a) the streaming pattern and (b) FOA averaged along the $x$ direction. Although the curves corresponding to different $t$ values follow similar profiles, inappropriate selection of $t$ can induce errors in the analysis. For example, in Fig. 1, at $t=0.14 \mathrm{~s}$, many of the $0.5-\mu \mathrm{m}$ particles have not entered quasiequilibrium, while some of the $5.0-\mu \mathrm{m}$ particles have already reached node lines and stopped moving at $t=0.56 \mathrm{~s}$.

\section{APPENDIX C: PARAMETERS IN PIV ANALYSIS}

In PIV calculations, each recorded image is split into many interrogation areas, while the size of each interrogation area and the overlapping rate between adjacent areas are important. Here, these two parameters are varied to obtain optimized results. The $x$-averaged $y$ component particle velocity profiles shown in Fig. 8 show that smoother velocity patterns can be observed with a larger overlapping rate and smaller size of individual interrogation areas. However, computational cost and noise level can also be more significant if the interrogation areas are too small [18]. As a trade-off, $96 \times 96$ pixels per interrogation area and $75 \%$ overlapping rate are chosen.

\section{APPENDIX D: WEIGHTING OF RADIATION FORCE AND STREAMING}

According to Eq. (1), the motion velocity of particles in acoustophoresis is a joint result of ARF and AS. By considering $\boldsymbol{v}_{p}$ as a weighted sum of contributions from ARF and AS, two weighting factors $\alpha$ (for ARF) and $\beta$ (for AS) (a)

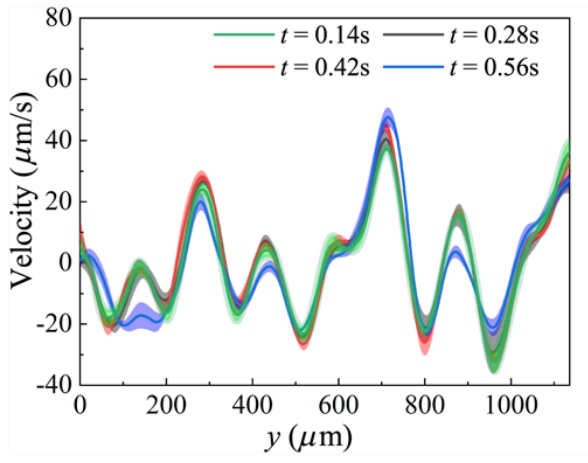

(b)

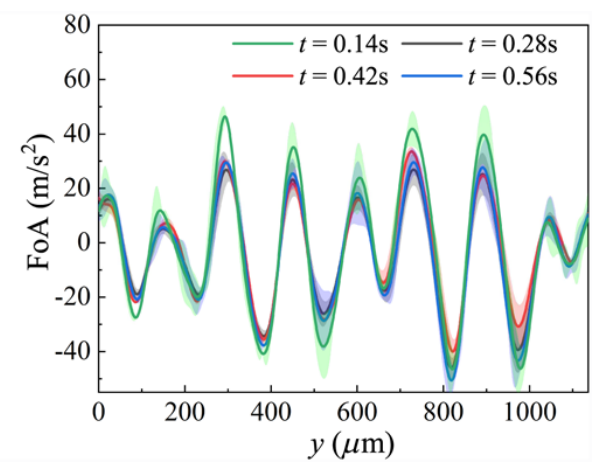

FIG. 7. The $x$-averaged $y$-component (a) $\mathrm{AS}$ and (b) FOA determined from $\mathrm{MR} \mu \mathrm{PIV}$ at different times. The shadowed areas indicate corresponding errors in three replicate measurements. 
(a)

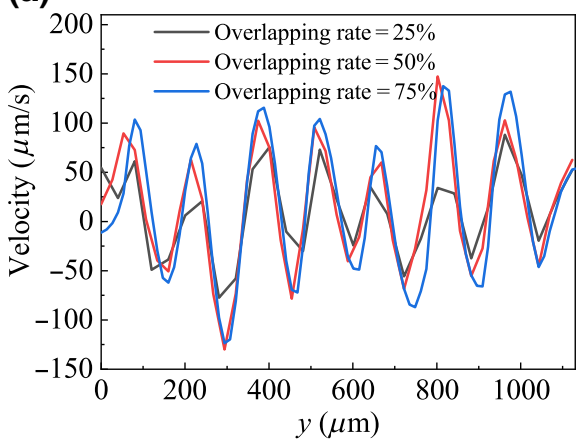

(b)

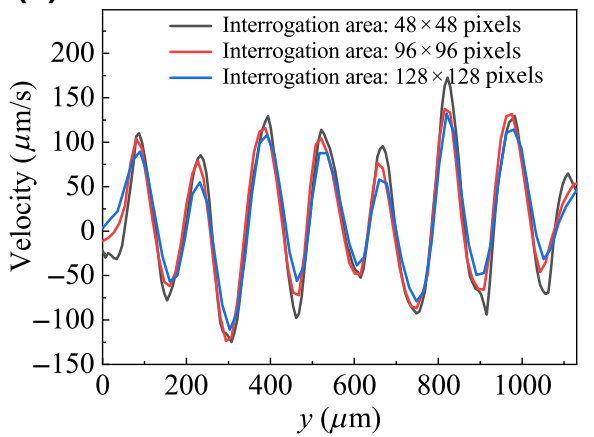

FIG. 8. The $x$-averaged $y$-component particle velocity profiles with varied (a) overlapping rate and (b) size of each individual interrogation area used in PIV analysis. are introduced, i.e.,

$$
\alpha r^{2}\left(\boldsymbol{a}_{r}-\boldsymbol{a}_{p}\right) / B+\beta \boldsymbol{v}_{m}=\boldsymbol{v}_{p}^{\prime} .
$$

For simplicity, coordinates $(x, y)$ are omitted in the field variables $\boldsymbol{a}_{r}(x, y) ; \boldsymbol{a}_{p}(x, y) ; \boldsymbol{v}_{m}(x, y)$; and $\boldsymbol{v}_{p}^{\prime}(x, y)$. By selecting $\alpha$ and $\beta$ properly, the difference between the estimated $\boldsymbol{v}_{p}^{\prime}$ and measured $\boldsymbol{v}_{p}$ can be minimized. The squared difference between them is

$$
E(\alpha, \beta)=\left(\boldsymbol{v}_{p}^{\prime}-\boldsymbol{v}_{p}\right)^{2} .
$$

For example, by considering the $x$-averaged $y$-component field velocity $v_{p, y}(y)$ and $v_{p, y}^{\prime}(y)$ on the right-hand side of Eq. (D2), the squared difference can be summed as

$$
\begin{aligned}
\Psi(\alpha, \beta) & =\sum_{y} E(\alpha, \beta) \\
& =\sum_{y}\left[\alpha R(y)+\beta v_{m, y}(y)-v_{p, y}(y)\right]^{2} .
\end{aligned}
$$

Here, $R(y)$ refers to the $x$-averaged $r^{2}\left(a_{r, y}-a_{p, y}\right) / B$. The weighting factors of contributions from ARF and AS can then be obtained with minimized $\Psi(\alpha, \beta)$, by requiring $\partial \Psi / \partial \alpha=\partial \Psi / \partial \beta=0, \quad\left(\partial^{2} \Psi / \partial \alpha \partial \beta\right)^{2}-\left(\partial^{2} \Psi / \partial \alpha^{2}\right)$ $\left(\partial^{2} \Psi / \partial \beta^{2}\right)<0$, and $\partial^{2} \Psi / \partial \alpha^{2}>0$.

\section{APPENDIX E: CALIBRATION OF THE ACOUSTIC PRESSURE AMPLITUDE}

To verify the measured $p_{0}$ and the EASF, a calibration procedure is carried out using microscopy and $\mathrm{FE}$ simulations combined with laser vibrometry.

First, with $z$ being the coordinate along the height of the channel, the channel bottom is located at $z_{1}=0$. By using a motorized microscope (IX-83, Olympus), most particles are found at $z_{2}=43 \pm 1 \mu \mathrm{m}$ after the acoustophoresis process is complete. Specifically, the microscope is first adjusted, so that several metal spots (with thickness 220 $\mathrm{nm}$ ) deposited on the substrate are clearly identified, and the position of the motorized stage is recorded as $z_{1}=0$. After the acoustic field is turned on, the focal plane is adjusted such that most particles can be observed clearly. After three repeated observations, the particles are confirmed to be located at $z_{2}=43 \mu \mathrm{m}$. According to Guo et $a l$., the particles should aggregate on an $x-y$ plane $\left(z_{3}=\right.$ $50 \mu \mathrm{m}$ in the current configuration) where they have maximum time-averaged kinetic energy (KE) [30]. Similar to the observations of Shi et al., the observed particle plane is a little lower than predicted, i.e., $z_{2}<z_{3}$, due to several factors [31].

Second, standing patterns also exist along the $z$ direction due to the reflective nature of the chamber ceiling, as is
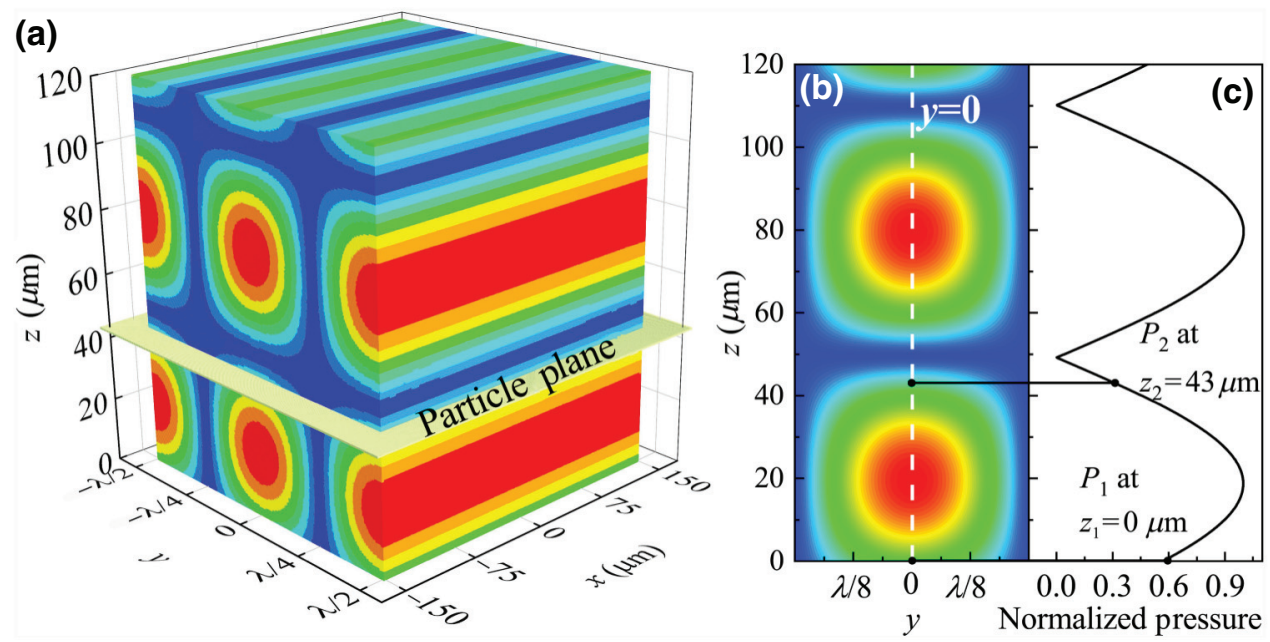

FIG. 9. (a) Normalized 3D sound pressure field. (b) The $y-z$ pattern of the acoustic pressure field at $x=0$ and (c) the corresponding pressure profile at $y=0$. 
indicated from the acoustic pressure field (Fig. 9) predicted through FE simulations using COMSOL MULTIPHYSICS (v5.2a, Comsol Inc.). With the profile of acoustic pressure amplitude (at $x=0$ and $y=0$ ) as a function of $z$ shown in Fig. 9(c), a scaling factor $\gamma=p_{0, P_{1}} / p_{0, P_{2}}=1.81$ is obtained between the pressure amplitudes at $P_{1}\left(z_{1}=0\right)$ and $P_{2}\left(z_{2}=43 \mu \mathrm{m}\right)$.

Finally, the $z$-component vibration velocity amplitude at $P_{1}, v_{0, P_{1}}$, is detected with a laser vibrometer (OFV-505, Polytech). In the measurement, a focused laser beam is used to scan the upper surface $\left(z_{1}=0\right)$ of the lithium niobate substrate across a $1 \times 1-\mathrm{mm}$ area, while the maximum amplitude of vibration velocity is recorded as $v_{0, P_{1}}$. The expected $p_{0}$ (at the particle plane) is then calculated as $p_{0, P_{2}}=p_{0, P_{1}} / \gamma=v_{0, P_{1}} \rho_{m} c_{m} / \gamma$, where $c_{m}=1483 \mathrm{~m} / \mathrm{s}$ is the sound speed of water.

[1] D. J. Collins, R. O’Rorke, C. Devendran, Z. Ma, J. Han, A. Neild, and Y. Ai, Self-Aligned Acoustofluidic Particle Focusing and Patterning in Microfluidic Channels from Channel-based Acoustic Waveguides, Phys. Rev. Lett. 120, 074502 (2018).

[2] M. Rajabi and A. Mojahed, Acoustic manipulation: Bessel beams and active carriers, Phys. Rev. E 96, 043001 (2017).

[3] K. W. Cushing, F. Garofalo, C. Magnusson, L. Ekblad, H. Bruus, and T. Laurell, Ultrasound characterization of microbead and cell suspensions by speed of sound measurements of neutrally buoyant samples, Anal. Chem. 89, 8917 (2017).

[4] N. I. Grigorchuk and P. M. Tomchuk, Force of optical radiation pressure on a spheroidal metallic nanoparticle near a plasmonresonance, Low Temp. Phys. 33, 851 (2007).

[5] T. B. Jones, in Electromechanics of Particles (Cambridge University Press, New York, 1995), Chap. 2, p. 11.

[6] H. Ebrahimian, M. Giesguth, K.-J. Dietz, G. Reiss, and S. Herth, Matnetic tweezers for manipulation of magnetic particles in single cells, Appl. Phys. Lett. 104, 063701 (2014).

[7] C. Devendran, D. J. Collins, Y. Ai, and A. Neild, HuygensFresnel Acoustic Interference and the Development of Robust Time-Averaged Patterns from Traveling Surface Acoustic Waves, Phys. Rev. Lett. 118, 154501 (2017).

[8] A. Anhäuser, R. Wunenburger, and E. Brasselet, Acoustic Rotational Manipulation using Orbital Angular Momentum Transfer, Phys. Rev. Lett. 109, 034301 (2012).

[9] J. T. Karlsen and H. Bruus, Acoustic Tweezing and Patterning of Concentration Fields in Microfluidics, Phys. Rev. Appl. 7, 034017 (2017).

[10] T. Lao, Z. Jiang, J. Yun, W. Qiu, F. Guo, C. Huang, J. D. Mancini, K. Gupta, M. E. Laucho-Contreras, Z. Z. C. Naing, L. Zhang, M. A. Perrella, C. A. Owen, E. K. Silverman, and X. Zhou, Hhip haploinsufficiency sensitizes mice to age-related emphysema, PNAS 113, E4681 (2016).

[11] M. Wu, Y. Ouyang, Z. Wang, R. Zhang, P.-H. Huang, C. Chen, H. Li, P. Li, D. Quinn, M. Dao, S. Suresh, Y. Sadovsky, and T. J. Huang, Isolation of exosomes from whole blood by integrating acoustics and microfluidics, PNAS 114, 10584 (2017).

[12] R. Barnkob, P. Augustsson, T. Laurell, and H. Bruus, Acoustic radiation- and streaming-induced microparticle velocities determined by microparticle image velocimetry in anultrasound symmetry plane, Phys. Rev. E 86, 056307 (2012).

[13] P. B. Muller and H. Bruus, Theoretical study of time-dependent, ultrasound-induced acoustic streaming in microchannels, Phys. Rev. E 92, 063018 (2015).

[14] P. G. Bassindale, D. B. Phillips, A. C. Barnes, and B. W. Drinkwater, Measurements of the force fields within an acoustic standing wave using holographic optical tweezers, Appl. Phys. Lett. 104, 163504 (2014).

[15] M. Wiklund, P. Spégel, S. Nilsson, and H. M. Hertz, Ultrasonic-trap-enhanced selectivity in capillary electrophoresis, Ultrasonics 41, 329 (2003).

[16] J. Hultström, O. Manneberg, K. Dopf, H. M. Hertz, H. Brismar, and M. Wiklund, Proliferation and viability of adherent cells manipulated by standing-wave ultrasound in a microfluidic chip, Ultrasound Med Biol 33, 145 (2007).

[17] K. Prestridge, P. Vorobieff, P. M. Rightley, and R. F. Benjamin, Validation of an Instability Growth Model using Particle Image Velocimetry Measurements, Phys. Rev. Lett. 84, 4353 (2000).

[18] P. Augustsson, R. Barnkob, S. T. Wereley, H. Bruus, and T. Laurell, Automated and temperature-controlled microPIV measurements enabling long-term-stable microchannel acoustophoresis characterization, Lab Chip 11, 4152 (2011).

[19] S. Liu, G. Xu, Z. Ni, X. Guo, L. Luo, J. Tu, and D. Zhang, Quantitative assessment of acoustic pressure in one-dimensional acoustofluidic devices driven by standing surface acoustic waves, Appl. Phys. Lett. 111, 043508 (2017).

[20] P. B. Muller, M. Rossi, A. G. Marin, R. Barnkob, P. Augustsson, T. Laurell, C. J. Kähler, and H. Bruus, Ultrasound-induced acoustophoretic motion of microparticles in three dimensions, Phys. Rev. E 88, 023006 (2013).

[21] R. Barnkob, C. J. Kähler, and M. Rossi, General defocusing particle tracking, Lab Chip 15, 3556 (2015).

[22] H. Bruus, Acoustofluidics 7: The acoustic radiation force on small particles, Lab Chip 12, 1014 (2012).

[23] C. Devendran, T. Albrecht, J. Brenker, T. Alan, and A. Neild, The importance of travelling wave components in standing surface acoustic wave (SSAW) systems, Lab Chip 16, 3756 (2016).

[24] P. B. Muller, R. Barnkob, M. J. H. Jensen, and H. Bruus, A numerical study of microparticle acoustophoresis driven by acoustic radiation forces and streaming-induced drag forces, Lab Chip 12, 4617 (2012).

[25] A. Lamprecht, S. Lakamper, T. Baasch, I. A. T. Schaap, and J. Dual, Imaging the position-dependent $3 \mathrm{~d}$ force on microbeads subjected to acoustic radiation forces and streaming, Lab Chip 16, 2682 (2016).

[26] J. F. Spengler, W. T. Coakley, and K. T. Christensen, Microstreaming effects on particle concentration in an ultrasonic standing wave, AIChE J. 49, 2773 (2003). 
[27] R. Barnkob, P. Augustsson, T. Laurell, and H. Bruus, Measuring the local pressure amplitude in microchannel acoustophoresis, Lab Chip 10, 563 (2010).

[28] B. P. Ho and L. G. Leal, Inertial migration of rigid spheres in two-dimensional unidirectional flows, J. Fluid Mech. 65, 365 (1974).

[29] S. M. Hagsäter, T. G. Jensen, H. Bruus, and J. P. Kutter, Acoustic resonances in microfluidic chips: Full-image micro-PIV experiments and numerical simulations, Lab Chip 7, 1336 (2007).
[30] F. Guo, Z. Mao, Y. Chen, Z. Xie, J. P. Lata, P. Li, L. Ren, J. Liu, J. Yang, M. Dao, S. Suresh, and T. J. Huang, Three-dimensional manipulation of single cells using surface acoustic waves, PNAS 113, 1522 (2016).

[31] J. Shi, S. Yazdi, S. C. Lin, X. Ding, I. K. Chiang, K. Sharp, and T. J. Huang, Three-dimensional continuous particle focusing in a microfluidic channel via standing surface acoustic waves (SSAW), Lab Chip 11, 2319 (2011). 